\title{
Consciência Situacional em Ambientes Educacionais como Apoio à Tomada de Decisão
}

\author{
Dirlene Silva ${ }^{1}$, Ernani Martins ${ }^{2}$, Luciana Assis ${ }^{1}$, Claudia Berti ${ }^{2}$, \\ Alessandro Vivas ${ }^{2}$, Cristiano Pitangui ${ }^{1}$ \\ ${ }^{1}$ Programa de Pós-Graduação em Educação - PPGEd \\ Universidade Federal dos Vales do Jequitinhonha e Mucuri (UFVJM) \\ Diamantina - MG - Brasil \\ ${ }^{2}$ Departamento de Computação \\ Universidade Federal dos Vales do Jequitinhonha e Mucuri (UFVJM) \\ Diamantina - MG - Brasil \\ \{dirlenesi, naninmartins, lupassis, claudiabberti, \\ alessandro.vivas,pitangui.cristiano\}@gmail.com
}

\begin{abstract}
Understanding the situation as a precursor of the decision-making process is a concept called Situational Awareness. This concept coupled with computational Artificial Intelligence techniques can be employed to mitigate the increasing rates of evasion in distance courses. This paper proposes a Decision Support Model in Virtual Educational Environments using Situational Consciousness and techniques of feeling analysis.
\end{abstract}

Resumo. A compreensão da situação como precursora do processo de Tomada de Decisão é um conceito denominado Consciência Situacional. Este conceito, aliado às técnicas computacionais de Inteligência Artificial, pode ser empregado para atenuar os crescentes índices de evasão em cursos à distância. Este trabalho propõe um Modelo De Apoio à Decisão em Ambientes Educacionais Virtuais utilizando Consciência Situacional e técnicas de Análise de Sentimento.

\section{Introdução}

Com o crescente número de cursos à distância, intensificou-se o uso de Ambientes Virtuais de Aprendizagem (AVA's) e, consequentemente, o uso das Tecnologias de Informação e Comunicação (TIC's) [da Cunha and da Silva 2009]. Tais tecnologias proporcionam às instituições novas possibilidades para o ensino e aprendizado, o que abre precedentes para a interatividade educacional [Azevedo et al. 2017].

Os AVA's contém diversas ferramentas que podem auxiliar o professor ou tutor em suas atividades cotidianas (controle de recebimento de atividades, maior iteração entre estudantes e professores, entrega e correção facilitada de trabalhos, entre outras) [da Cunha and da Silva 2009]. Uma ferramenta que exemplifica novas tecnologias utilizadas para complementar os métodos tradicionais na Educação a Distância (EaD) são os fóruns de discussão on-line [Belarmino Rolim et al. 2017].

Os fóruns são muito utilizados por permitirem discussões simultâneas de um ou diversos conteúdos disponibilizados entre estudantes e professores, sem barreiras temporais ou geográficas, proporcionando maior flexibilidade na programação de estudo. Estudos como o realizado em [Cheng et al. 2011] demonstram que a participação em fóruns, 
VIII Congresso Brasileiro de Informática na Educação (CBIE 2019)

Anais do XXX Simpósio Brasileiro de Informática na Educação (SBIE 2019)

particularmente a leitura de posts nos cursos à distância, tendem a melhorar o desempenho do estudante no curso.

Portanto, o fórum é uma ferramenta importante de apoio ao trabalho em um AVA. Todavia, a grande quantidade de postagens gera uma dificuldade para o professor em controlar e acompanhar, de forma individualizada e em tempo hábil, as mensagens geradas pelos estudantes [Belarmino Rolim et al. 2017]. Apesar das vantagens em se utilizar os AVA's, o número de evasões é crescente e preocupante para professores, tutores e instituições de ensino, que têm como uma das metas atenuar os índices de evasão dos cursos por parte dos estudantes [Manhães et al. 2011]. Desta forma, propostas de desenvolvimento de ferramentas que tratam este problema se fazem necessárias.

Devido ao excesso de atividades desempenhadas por professores ou tutores em AVA's, o fato de um determinado estudante não estar envolvido de forma adequada no curso pode ser imperceptível. Por ser o ambiente de ensino complexo e dinâmico, garantir a percepção de elementos relevantes à sua identificação é algo nem sempre trivial [Berti et al. 2017].

Técnicas da Análise de Sentimento têm sido utilizadas para obtenção de informações que indiquem o sentimento e pensamento do estudante [Cambria 2016]. Como os fóruns geram maior interatividade entre estudantes e professores, estes podem, aliados às técnicas computacionais da Análise de Sentimento, auxiliar professores e tutores na Tomada de Decisão frente às possíveis evasões. A correta compreensão das situações que são desenvolvidas entre professor/tutor e estudante, ocorridas em um fórum de discussão, pode apoiar sistemas que atendam com maior eficácia o processo de aprendizagem educacional [Azevedo et al. 2017].

A interação entre professores e estudantes na $\mathrm{EaD}$ é relevante para manutenção do interesse desses estudantes. Desta forma, é necessário que professores e/ou tutores mantenham compreensão da situação que envolve as reações e as demonstrações dos sentimentos dos estudantes, frequentemente expressados nos fóruns [Cheng et al. 2011]. A compreensão dos elementos do ambiente e suas relações e a projeção do seu estado futuro é denominado Consciência Situacional - CS, do inglês Situation Awareness - SAW [Endsley and Jones 2012].

Técnicas baseadas em Análise de Sentimento e Consciência Situacional podem auxiliar o entendimento do ambiente educacional, possibilitando identificar precocemente o estudante propenso a evadir, auxiliando professores ou tutores na Tomada de Decisão. Não é recorrente o uso de modelos computacionais baseados em CS aplicados em AVA's. Sendo assim, este trabalho propõe a construção de um modelo de Tomada de Decisão baseado em CS apoiado por técnicas da Análise de Sentimento para dar suporte ao professor no ambiente de EaD. A Análise de Sentimento é uma ferramenta utilizada neste trabalho conjuntamente ao conceito de Consciência Situacional. No entanto, o modelo suporta outras técnicas e métodos computacionais.

\section{Fundamentação teórica}

Nesta seção são conceituados Análise de Sentimento e Consciência Situacional, que fundamentam o modelo de apoio a Tomada de Decisão em AVA's. 
VIII Congresso Brasileiro de Informática na Educação (CBIE 2019)

Anais do XXX Simpósio Brasileiro de Informática na Educação (SBIE 2019)

\subsection{Consciência Situacional}

CS é a compreensão do que está acontecendo ao redor, e do que esta informação pode significar no momento presente e no futuro próximo. É um componente natural da cognição humana e sua importância nos processos decisórios e estratégicos passou a ser reconhecida e identificada por um piloto da Primeira Guerra Mundial chamado Oswald Boekle que declarou a importância de "ganhar a consciência do inimigo antes que o inimigo ganhe uma consciência similar" e desenvolveu métodos para realizá-la [Jeannot 2000].

A CS é um elemento essencial e precursor na Tomada de Decisão individual ou coletiva. A forma como alguém compreende o ambiente ao seu redor constitui a sua CS, baseado nos elementos deste ambiente, na compreensão da relação destes elementos associada ao seu Modelo Mental sobre a situação. Aprimorar a CS de uma equipe pode ajudar a consolidar decisões e estratégias mais apropriadas à situação em curso [Silva et al. 2012].

O termo CS, apesar de ter sido inicialmente utilizado na área militar, atualmente está presente em processos decisórios das mais variadas áreas como, por exemplo, médica, resposta a emergências, esportes, navegação, gerenciamento de crises, controle de tráfego aéreo, dentre outras. Em alguns casos, como por exemplo para controladores de tráfego aéreo, a CS é referenciada como uma Representação Mental da situação na qual as decisões são baseadas [Endsley and Jones 2012]. Representação Mental, Modelo Mental ou Imagem Mental são as informações captadas pela mente humana sobre o mundo exterior [Moreira 1996]. A CS é composta por três níveis: (1) a percepção dos elementos no ambiente dentro de um volume de tempo e espaço, (2) a compreensão de seu significado e a (3) projeção de seu estado em um futuro próximo [Endsley and Jones 2012].

A capacidade de uma pessoa perceber diversas informações simultaneamente é limitada. Esta limitação interfere na capacidade de processamento de tais informações, criando um dos grandes incentivos para a construção de sistemas de apoio à Tomada de Decisão fundamentados na CS. As informações captadas pelas pessoas ficam armazenadas na memória e esta pode ser classificada como de longo prazo (mantem informações por um longo período de tempo) ou de trabalho (também conhecida por memória de curto prazo, e armazena elementos por um tempo limitado). As memórias de trabalho e de longo prazo, desempenham papéis essenciais para ajudar a alcançar a CS [Endsley and Jones 2012].

Apesar de sua limitação, a memória de trabalho é essencial na Tomada de Decisão e forma o segundo maior gargalo para a CS. A informação recente, presente na memória de trabalho, é conciliada com conhecimentos, presentes na memória de longo prazo, criando assim uma nova Imagem Mental ou atualizando uma mudança da situação. Estes fragmentos de informação são processados e utilizados para criar possíveis projeções do futuro. As projeções criadas irão auxiliar o indivíduo a decidir que ações tomar para alcançar resultados condizentes com seus objetivos. A memória de longo prazo estrutura o conhecimento como esquemas e modelos mentais [Endsley and Jones 2012].

Modelos mentais dependem da habilidade e aprendizado do indivíduo adquiridos ao longo do tempo. Além disso, desempenham um papel significativo na melhoria da CS do indivíduo [Endsley and Jones 2012]. Este conceito fundamenta o uso de Aprendizado Supervisionado que compõe a proposta deste trabalho e será apresentado na seção 2.2. 
VIII Congresso Brasileiro de Informática na Educação (CBIE 2019)

Anais do XXX Simpósio Brasileiro de Informática na Educação (SBIE 2019)

A Figura 1 ilustra, de forma simplificada, a relação do Modelo Mental e o processo de Consciência Situacional para Tomada de Decisão. Modelos Mentais possuem papel crucial no desenvolvimento e manutenção de CS [Berti et al. 2017].

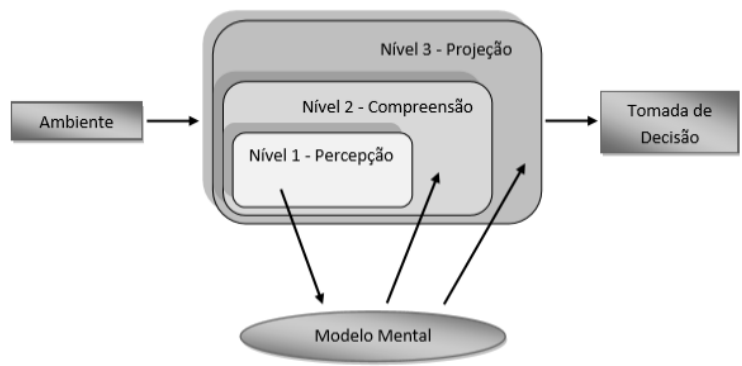

\section{Figura 1. Relação entre Modelo Mental e Consciência Situacional - Adaptado de [Endsley and Jones 2012]}

[Endsley and Jones 2012] descrevem que modelos mentais "são estruturas complexas que as pessoas usam para modelar o comportamento de sistemas específicos. É a compreensão sistemática de como algo funciona”. Para [Moreira 1996], não existe um único Modelo Mental para um determinado conceito, "são uma forma de representação analógica do conhecimento". E ainda acrescenta que "são o que as pessoas realmente tem em suas cabeças e o que guia o uso que fazem das coisas". Modelos mentais são gerados a partir de um conhecimento prévio e servem para ajudar a pessoa a determinar quais informações são importantes para serem atendidas e ajudam na compreensão dos níveis 2 e 3 da CS [Endsley and Jones 2012].

Como os modelos mentais são construídos a partir de elementos básicos organizados de forma estruturada para representar determinado conceito, eles podem ser computáveis, ou seja, descritos na forma de procedimentos que possam ser executados por máquinas [Moreira 1996]. Desta maneira, modelos mentais podem ser apoiados por Análise de Sentimento para detectarem sinais que irão auxiliar na prevenção da evasão escolar. Para tanto, Algoritmos de Aprendizado de Máquina são utilizados como modelos mentais, visto que é preciso um aprendizado prévio e, posteriormente a aplicação dos algoritmos para projetar um estado futuro (no caso, uma provável evasão), e intervir antecipadamente.

\subsection{Análise de Sentimento}

A Análise de Sentimento (AS) ou Mineração de Opinião, é um campo da Inteligência Artificial (IA) que visa identificar, extrair e analisar o sentimento, a emoção ou opinião que os usuários apresentam a respeito de alguma entidade de interesse (um produto, uma empresa, um lugar, um conteúdo, dentre outras) baseado no conteúdo disponível na web e categorizar a satisfação identificada, classificando-os de acordo com sua polaridade, ou seja, como positivos ou negativos [Kauer 2016]. Tem como principal objetivo obter relatórios da opinião das pessoas acerca de algum item, sem precisar ter acesso a todas as opiniões e notícias a respeito.

A AS possui pesquisas relacionadas ao Processamento de Linguagem Natural (PLN), com aplicações em várias áreas como: educação [da Cunha and da Silva 2009], desenvolvimento de técnicas de detecção e sumarização automática de opiniões sobre produtos e serviços [da Silva et al. 2017], uso de mecanismos de busca, análise de 
VIII Congresso Brasileiro de Informática na Educação (CBIE 2019)

Anais do XXX Simpósio Brasileiro de Informática na Educação (SBIE 2019)

opiniões, sentimentos, emoções e atitudes de pessoas com utilização de mídias sociais [Kauer 2016] [Cambria 2016], na saúde, em monitoração de perturbações psicológicas e detecção de depressão [Zucco et al. 2017], aplicações de inteligência governamentais [Cambria 2016], dentre outras.

No meio educacional, a AS pode ser usada para mensurar a motivação dos estudantes a partir de interações em fóruns educacionais [da Cunha and da Silva 2009]. Entender as reações do estudante usando a classificação de polaridade, ou seja, identificar se o estudante encontra-se motivado ou desmotivado, pode ser um caminho para se encontrar os motivos (ou indícios) da evasão. A criação de sistemas que envolvam, além da automação do processo de aprendizagem, a interpretação e compreensão do sentimento do estudante, pode auxiliar os gestores e tutores no processo de Tomada de Decisão para ações efetivas para prevenção da evasão.

O processo de AS pode ser caracterizado basicamente em três etapas: 1) Coleta de conteúdos: etapa em que acontece a busca em fontes diversas, como comentários, anúncios, documentos, dentre outros; 2) Classificação de polaridade: etapa considerada a mais importante, pois é onde ocorre a identificação da polaridade ou orientação da opinião do texto. Existem diversas técnicas de classificação, e entre elas se encontram o Aprendizado de Máquina, a Seleção de Palavras e a Análise Sintática; e 3) Sumarização dos resultados: nesta etapa os resultados são apresentados e podem ser de forma textual, tabelas ou gráficos [Becker and Tumitan 2013].

Para a etapa de Classificação, que é o foco deste trabalho, diferentes abordagens são propostas na literatura, podendo ser usadas de forma isolada ou em combinação para melhoria nos resultados, em que algumas se destacam a) Léxicas, com uso de dicionários de sentimentos; b) Aprendizado de Máquina, com predominância no uso de técnicas de classificação ou regressão; c) Estatísticas, com uso de técnicas para avaliar a ocorrência de termos; e d) Semânticas, fazendo comparação entre a polaridade encontrada com outras de polaridade conhecida [Becker and Tumitan 2013].

O uso de Dicionário de Sentimentos e o Aprendizado de Máquina são as duas abordagens mais utilizadas e que possuem melhores desempenhos [Souza et al. 2011]. Neste trabalho foi utilizado o dicionário de opiniões [Kauer 2016] Oplexicon 3.0, que é específico para a língua portuguesa do Brasil e classifica as palavras em valores numéricos de acordo com o sentimento que remete [Freitas et al. 2015].

A aplicação de algoritmos de Aprendizado de Máquina tem como objetivo descobrir de forma automática regras gerais em grandes conjuntos de dados, que permitam extrair informações implicitamente representadas [Becker and Tumitan 2013]. Esta técnica pode ser divida em dois tipos: Aprendizado Supervisionado e Aprendizado Não Supervisionado.

O Método Supervisionado possui predominância na área de mineração de opiniões [Becker and Tumitan 2013] e consiste em obter a base de dados de fatos ocorridos e, em seguida, dividi-la. Uma das partes é reservada para treinamento previamente rotulada com as classes consideradas (e.g. positivo, negativo, neutra), e posteriormente a outra parte é colocada para teste a fim de avaliar a frequência de ocorrência das palavras [Cambria 2016].

Em ambientes educacionais a Análise de Sentimento e a utilização de métodos 
VIII Congresso Brasileiro de Informática na Educação (CBIE 2019)

Anais do XXX Simpósio Brasileiro de Informática na Educação (SBIE 2019)

e técnicas de Consciência Situacional são promitentes como caminhos para reduzir os índices da evasão, visto que a correta compreensão da situação que ocorre no ambiente é a precursora da Tomada de Decisão.

\section{Modelo de Apoio a Decisão em Ambientes Educacionais Virtuais}

Este trabalho propõe a construção de um modelo, utilizando Consciência Situacional e Análise de Sentimento, para apoiar o processo de Tomada de Decisão de professores ou tutores em AVA's. O modelo (Figura 2) visa sistematizar a implementação de sistemas sustentado por CS do ambiente em AVA's, e é constituído de três etapas: (1) Ambiente de Ensino, (2) Motor Situacional e (3) Consciência Situacional.

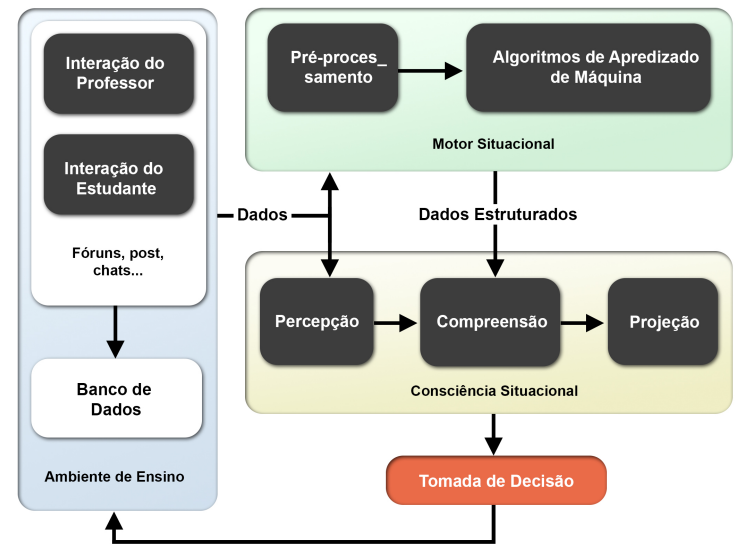

Figura 2. Modelo Conceitual de Apoio à Tomada de Decisão em AVA's sustentado por Consciência Situacional. Fonte: Elaborado pelo autor

Como primeiro passo na aquisição da CS, a percepção consiste em notar os sinais do ambiente, e desempenha papel primordial na obtenção dos dados. As fontes de dados a serem consideradas no modelo conceitual são obtidas da interação online entre professores e estudantes através das seguintes ferramentas: fóruns, chat, e-mail, posts, wikis, dentre outras. Todo o material disponibilizado para os estudantes, e também as discussões ocorridas acerca do conteúdo trabalhado, são armazenados em banco de dados. Quanto mais interação houver entre professores e estudantes, maiores serão as fontes de dados que alimentam o modelo consciente de situação no Ambiente de Ensino.

O modelo apresentado é genérico e pode utilizar diferentes informações presentes no Ambiente de Ensino. Para o desenvolvimento deste trabalho, as fontes de dados utilizadas foram as mensagens (textos livres) contidas nos fóruns e estes serviram como dados de entrada para o Motor Situacional. O Motor Situacional age como o papel dos esquemas e roteiros do Modelo Mental do professor ou tutor (considerados como operadores em um ambiente educacional), e é composto pelo Pré-Processamento e pelos Algoritmos de Aprendizado de Máquina.

Como os dados obtidos e armazenados no ambiente de ensino vem crescendo em grande volume nos últimos anos, em especial os dados textuais [Azevedo et al. 2017] e estes são inseridos sem tratamento, é necessário uma etapa de pré-processamento. Nesta fase, os dados e informações serão estruturados para se adequarem às bases de dados que a ferramenta utilizará ou até mesmo à linguagem de programação adotada. A etapa de 
VIII Congresso Brasileiro de Informática na Educação (CBIE 2019)

Anais do XXX Simpósio Brasileiro de Informática na Educação (SBIE 2019)

pré-processamento é considerada uma das mais importantes, visto que todas as palavras que agregam pouco ou nenhum valor ao texto (como, artigos, preposições e pronomes) são retiradas.

A fase de pré-processamento deve ser prevista com o objetivo de adequar e estruturar os dados para que possam ser utilizados como entrada para os Algoritmos de Aprendizado de Máquina. Os Algoritmos de Aprendizado de Máquina são utilizados para a formalização ou identificação da situação. Os modelos preditivos (dados ou informações) que são gerados pelos algoritmos utilizados neste passo, ao serem armazenados, servirão de Modelos Mentais para os sistemas computacionais de apoio a Tomada de Decisão, ou seja, um esquema arquivado para consulta e imediata identificação da situação, promovendo a compreensão e projeção da situação e consequentemente a CS.

A Tomada de Decisão é o resultado final do processo. É a última etapa do modelo conceitual e apresenta a alternativa determinada pelo operador diante das opções suportadas pelo sistema. Toda decisão impactará em um nova configuração do Ambiente de Ensino, criando assim novos parâmetros e situações em uma futura iteração.

\section{Testes e Análise dos Resultados}

Esta seção apresenta um estudo de caso que possibilita demonstrar a aplicação computacional do modelo proposto. Inicialmente, é descrita a base de dados utilizada; então, são apresentadas as técnicas de pré-processamento e os algoritmos aplicados no processo de construção dos Modelos Mentais, conforme o Motor Situacional representado na Figura 2. O objetivo dos experimentos foi de sistematizar a implementação de ferramentas, que, integradas a sistemas existentes, possam contribuir para adquirir e/ou prover a manutenção da Consciência Situacional do professor ou tutor em sistemas de apoio à Tomada de Decisão em ambientes de Educação a Distância.

\subsection{Caracterização da Base de Dados}

A base de dados utilizada neste trabalho é a BD1 descrita em [Azevedo et al. 2017], que foi obtida a partir de postagens inseridas em um fórum educacional pelos estudantes da disciplina Tópicos Avançados em Inteligência Artificial. Esta base é composta por 228 postagens (textos livres) rotuladas como pertencentes a estudantes motivados (107 postagens) e desmotivados (121 postagens).

\subsection{Pré-processamento da Base de Dados}

O pré-processamento é a fase iniciada após a criação ou aquisição da base de dados; é responsável por tratar os dados de forma que possam ser melhor trabalhados e para que as técnicas computacionais possam ser aplicadas [Belarmino Rolim et al. 2017]. Neste trabalho o pré-processamento foi dividido em quatro etapas.

$\mathrm{Na} 1^{\mathrm{a}}$ etapa foi feita a remoção de stopwords, ou seja, foram retiradas as palavras que acrescentam pouco ou nenhum significado ao texto. A $2^{\mathrm{a}}$ etapa foi a utilização da técnica de stemming, ou seja, redução da dimensionalidade dos dados, transformando palavras em seus radicais. Ao reduzir a dimensionalidade dos dados há uma tendência em melhorar a taxa de acerto dos algoritmos.

$\mathrm{Na} 3^{\mathrm{a}}$ etapa, foi gerada uma lista (vetor de características) com todas as palavras (atributos) presentes nas frases e atribuído a cada atributo 0 e 1 , onde 0 representa que $o$ 
VIII Congresso Brasileiro de Informática na Educação (CBIE 2019)

Anais do XXX Simpósio Brasileiro de Informática na Educação (SBIE 2019)

atributo não está presente e 1 que está presente na frase. Após essas duas etapas, a base de dados ficou com 1130 atributos (palavras únicas). A $4^{\mathrm{a}}$ etapa foi preparar a base de dados com objetivo de utilizá-la com o WEKA ${ }^{1}$.

\subsection{Aplicação dos Algoritmos do Aprendizado de Máquina}

Foram realizados experimentos utilizando a base de dados pré-processada com alguns algoritmos de aprendizagem supervisionada: Naive Bayes, Redes Bayesianas, Rede Neural MLP, SMO (Sequencial Minimal Optimization), C4.5, Linear Logistic Regression (Simple Logistic) e Random Forest. Os testes foram realizados utilizando uma abordagem de validação cruzada $k$-fold de 10 partições $(k=10)$ com objetivo de se obter uma métrica de classificação mais fiel.

A aplicação dos algoritmos é capaz de gerar modelos preditivos que atuam como esquemas do Modelo Mental do agente humano, direcionando para a compreensão e projeção da situação de maneira rápida, analogamente aos esquemas e roteiros do Modelo Mental. Compreensão e projeção da situação ocorrendo de maneira rápida, podem gerar Tomadas de Decisão igualmente rápidas [Berti et al. 2017]. Esse aspecto representa uma particularidade importante, devido ao tempo de resposta ser fator crucial, e até mesmo determinante para se ter um resultado satisfatório em ambientes educacionais.

Os experimentos descritos na Tabela 1 apresentam as médias das acurácias dos algoritmos em 30 execuções sobre a base de dados. Foram realizados dois tipos de experimentos: (1) Foi feito o pré-processamento somente com a Remoção de stopwords. Após essa etapa, a base de dados ficou com 1130 atributos (palavras únicas). (2) Foi feita a Remoção de stopwords e redução das palavras aos seus radicais (stemming). Neste caso a dimensão dos dados foi reduzida a 769 atributos. A métrica utilizada em ambos os casos foi a taxa de acerto (acurácia).

Tabela 1. Testes realizados usando Algoritmos de Aprendizado de Máquina Supervisionado, em uma abordagem de Validação Cruzada 10-folds

\begin{tabular}{|c|l|l|}
\hline Algoritmo & $\begin{array}{l}\text { Acurácia } \\
\text { (Stopwords) }\end{array}$ & $\begin{array}{l}\text { Acurácia } \\
\text { (Stopwords } \\
\text { + Stemmer) }\end{array}$ \\
\hline Naive Bayes & $76,09 \%$ & $75,65 \%$ \\
\hline Redes Bayesianas & $50,87 \%$ & $58,69 \%$ \\
\hline Rede Neural MLP & $66,09 \%$ & $77,82 \%$ \\
\hline SMO (Sequencial Minimal Optimization) & $75,65 \%$ & $76,52 \%$ \\
\hline C4.5 & $69,13 \%$ & $73,91 \%$ \\
\hline Linear Logistic Regression (Simple Logistic) & $73,04 \%$ & $76,95 \%$ \\
\hline Random Forest & $75,65 \%$ & $78,69 \%$ \\
\hline
\end{tabular}

Percebeu-se que, dentre os algoritmos analisados, o pior desempenho ficou com as Redes Bayesianas. Entre os demais algoritmos não houve diferença significativa, conforme aplicação do teste t-pareado (corrigido) com nível de significância de 5\%. Também

\footnotetext{
${ }^{1}$ Um software que contém uma coleção de algoritmos de Aprendizado de Máquina e é amplamente utilizado em tarefas de mineração de dados [Kauer 2016]. Disponível em: $<$ https : //www.cs.waikato.ac.nz/ml/weka/downloading.html >
} 
VIII Congresso Brasileiro de Informática na Educação (CBIE 2019)

Anais do XXX Simpósio Brasileiro de Informática na Educação (SBIE 2019)

foi possível notar que o uso da técnica de stemming trouxe uma melhoria na taxa de acerto dos algoritmos quando comparado ao pré-processamento que apenas removia stopwords.

A aplicação destes algoritmos mostrou que é possível obter taxas de acerto maiores que $78 \%$. Isto mostra a viabilidade de suas aplicações como parte do motor situacional do modelo proposto para auxiliar na Tomada de Decisão em ambientes educacionais.

Além dos testes mencionados, foram feitos testes com o dicionário de sentimentos Oplexicon, utilizando a linguagem de programação Python e a biblioteca de processamento de linguagem natural NLTK ${ }^{2}$. O método utilizado foi o Bag of Words [Wallach 2006] em que a classificação foi feita considerando o somatório da frequência das palavras, conforme as suas polaridades. Os resultados obtidos (55\%) com este método não foram satisfatórios. Logo, percebe-se que o dicionário utilizado no PLN não se ajusta bem aos textos dos fóruns de discussão utilizados neste trabalho. As técnica de aprendizado supervisionado apresentaram melhor desempenho, e, além disso, não dependem de um dicionário para fazer a Análise de Sentimento.

\section{Considerações finais}

O presente trabalho teve como objetivo apresentar um Modelo Conceitual de apoio à Tomada de Decisão em AVA's, sustentado por Consciência Situacional. Uma das principais vantagens obtidas pelo uso de sistemas conscientes à situação é o auxílio na compreensão da realidade, sendo possível uma visão aprimorada do ambiente em análise e a adaptação à sistemas computacionais.

O modelo apresentado é genérico e pode utilizar diferentes informações presentes no Ambiente de Ensino. Pelos experimentos realizados, pôde-se perceber que é viável utilizar algoritmos de Aprendizado de Máquina Supervisionado como sendo Motor Situacional em ambientes computacionais. Vale ressaltar que os resultados dos algoritmos não são a palavra final, ao se tratar de Tomada de Decisão. A experiência do operador, personalidade e características pessoais influenciam em todo processo decisório, visto que Consciência Situacional é uma condição individual. Fornecer ferramentas que possam auxiliar este processo pode contribuir para que o resultado do processo de Tomada de Decisão como um todo gere ações mais assertivas.

Trabalhos futuros podem: (i) Explorar a aplicação de outros algoritmos de Aprendizado de Máquina com outras ferramentas existentes em AVA's; (ii) Utilizar conjuntos de dados reais com diferentes tamanhos, quantidades de classes e dimensões, de cursos específicos da EaD.

\section{Referências}

Azevedo, D., Ferreira, R., Mendonca, V., and Miranda, P. (2017). Aplicação de análise de sentimento em fóruns educacionais para prevenir evasão. In Brazilian Symposium on Computers in Education (Simpósio Brasileiro de Informática na Educação-SBIE), volume 28, page 1097.

Becker, K. and Tumitan, D. (2013). Introdução à mineração de opiniões: Conceitos, aplicações e desafios. Simpósio brasileiro de banco de dados, 75.

\footnotetext{
${ }^{2}$ Natural Language Toolkit - Biblioteca da linguagem Python para trabalhar com dados de Processamento de Linguagem Natural. Disponível em: < https : //www.nltk.org/ >
} 
VIII Congresso Brasileiro de Informática na Educação (CBIE 2019)

Anais do XXX Simpósio Brasileiro de Informática na Educação (SBIE 2019)

Belarmino Rolim, V., de Mello, R. F. L., and de Barros Costa, E. (2017). Utilização de técnicas de aprendizado de máquina para acompanhamento de fóruns educacionais. Revista Brasileira de Informática na Educação, 25(3).

Berti, C. B. et al. (2017). Modelo preditivo de situações como apoio à consciência situacional e ao processo decisório em sistemas de resposta à emergência. $\mathrm{PhD}$ thesis, Tese (Doutorado)-Universidade Federal de São Carlos.

Cambria, E. (2016). Affective computing and sentiment analysis. IEEE Intelligent Systems, 31(2):102-107.

Cheng, C. K., Paré, D. E., Collimore, L.-M., and Joordens, S. (2011). Assessing the effectiveness of a voluntary online discussion forum on improving students' course performance. Computers \& Education, 56(1):253-261.

da Cunha, F. O. and da Silva, J. M. C. (2009). Análise das dimensões afetivas do tutor em turmas de ead no ambiente virtual moodle. In Brazilian Symposium on Computers in Education (Simpósio Brasileiro de Informática na Educação-SBIE), volume 1.

da Silva, L. K., Barbosa, M. L. K., Pandolfi, R., and Cazella, S. C. (2017). Análise de sentimento pela ótica da abordagem multimodal. RENOTE, 15(1).

Endsley, M. R. and Jones, D. G. (2012). Designing for Situation Awareness: An Approach to User-Centered Design. CRC Press.

Freitas, L. A. d. et al. (2015). Feature-level sentiment analysis applied to brazilian portuguese reviews. Tese (Pós-Doutorado)-Universidade Católica do Rio Grande do Sul.

Jeannot, E. (2000). Situation awareness, synthesis of literature research. EEC note, 16(00).

Kauer, A. U. (2016). Análise de sentimentos baseada em aspectos e atribuições de polaridade. Dissertação (Mestrado)-Universidade Federal de São Carlos.

Manhães, L. M. B., Da Cruz, S. M. S., Costa, R. J. M., Zavaleta, J., and Zimbrão, G. (2011). Previsão de estudantes com risco de evasão utilizando técnicas de mineração de dados. In Brazilian Symposium on Computers in Education (Simpósio Brasileiro de Informática na Educação-SBIE), volume 1.

Moreira, M. A. (1996). Modelos mentais. Investigações em ensino de ciências. Porto Alegre. Vol. 1, n. 3 (dez. 1996), p. 193-232.

Silva, A. W. L., Lacerda, M. R. M., Dos Santos, N., Fialho, F. A. P., and Netto, M. (2012). Consciência da situação em equipes transdisciplinares. Ciências \& Cognição, 17(2).

Souza, M., Vieira, R., Busetti, D., Chishman, R., and Alves, I. M. (2011). Construction of a portuguese opinion lexicon from multiple resources. In Proceedings of the 8th Brazilian Symposium in Information and Human Language Technology.

Wallach, H. M. (2006). Topic modeling: beyond bag-of-words. In Proceedings of the 23rd international conference on Machine learning, pages 977-984. ACM.

Zucco, C., Calabrese, B., and Cannataro, M. (2017). Sentiment analysis and affective computing for depression monitoring. In 2017 IEEE International Conference on Bioinformatics and Biomedicine (BIBM), pages 1988-1995. IEEE. 